[0212-7199(2002) 19: 1; pp 19-22] ANALES DE MEDICINA INTERN Copyright $\odot 2002$ ARAN EDICIONES, S.L.

AN. MED. INTERNA (Madrid) Vol. 19, N. $^{\circ} 1$, pp. $19-22,2002$

\section{Insuficiencia suprarrenal aguda por hemorragia suprarrenal bilateral como primera manifestación de un síndrome antifosfolípido. A propósito de un caso y revisión}

\author{
G. BARREIRO GARCÍA, M. V. EGURBIDE ARBERAS, A. ÁLVAREZ BLANCO, \\ J. UGALDE ESPIÑEIRA, C. AGUIRRE ERRASTI \\ Servicio de Medicina Interna. Hospital de Cruces. Baracaldo. Vicaya. Cátedra de \\ Patología Médica. Universidad del País Vasco
}

ACUTE ADRENAL INSUFICIENCY CAUSED BY ADRENAL HEMOR RHAGE AS A FIRST MANIFESTATION OF THE ANTIPHOSPHOLIPID SYNDROME. A CASE REPORT AND REVIEW

\begin{abstract}
RESUMEN
La hemorragia suprarrenal (HS) asociada a síndrome antifosfolípido (SAF) en relación con lupus eritematoso sistémico (LES), es poco frecuente. Puede ser la manifestación inicial de la enfermedad. Se invoca como patogenia la trombosis en las venas de drenaje suprarrenales e infarto hemorrágico secundario. El riesgo de trombosis recurrente en estos pacientes es alto. Se aconseja por ello la anticoagulación indefinida.
\end{abstract}

PALABRAS CLAVE: Hemorragia suprarrenal. Lupus eritematoso sistémico. Síndrome antifosfolípido. Anticoagulación.

\begin{abstract}
Although adrenal hemorrhage is an unusual feature of antiphospho lipid syndrome (APS), it can be its first clinical manifestation. The patho genetic substratum is the thrombosis of the adrenal veins leading to hemorrhagic infarction. Recurrences are frequent in patients with APSrelated thrombosis, thus long-term anticoagulation is advocated by most authors.
\end{abstract}

KEY WORDS: Adrenal hemorrhage. Systemic lupus erythematosus. Antiphospholipid syndrome. Anticoagulant therapy.

Barreiro García G, Egurbide Arberas MV, Álvarez Blanco A, Ugalde Espiñeira J, Aguirre Errasti C. Insuficiencia suprarrenal aguda por hemorragia suprarrenal bilateral como primera manifestación de un síndrome antifosfolípido. A propósito de un caso y revisión. An Med Interna (Madrid) 2002; 19: 19-22.

\section{INTRODUCCIÓN}

En estudios efectuados en autopsias la incidencia global de hemorragia suprarrenal (HS) es de 0,14-1,8\% (1). En una serie de 7.500 autopsias se presenta HS en $0,33 \%$, pero si se seleccionan los pacientes fallecidos por shock este porcentaje sube al 15,4\% (2). Esto lleva a decir al algunos autores que la HS es muy frecuente en pacientes graves, y que algunos de ellos pueden recuperados con un diagnóstico precoz (3). La HS asociada a síndrome antifosfolipídico (SAF), en relación o no a lupus eritematoso sistémico (LES), es una entidad poco frecuente. En raras ocasiones es la primera manifestación de la enfermedad (4). Actualmente es una causa conocida de insuficiencia suprarrenal a considerar en el estudio etiológico de las alteraciones suprarrenales (5).

\section{CASO APORTADO}

Mujer de 59 años con historia previa de poliartritis en metacarpofalángicas e interfalángicas proximales de ambas manos hace años, fotosensibilidad y fenómeno de Raynaud bilateral. Ingresa en el servicio de
Traumatología por presentar fractura de cadera izquierda subcapital tras caída casual, siendo intervenida 5 días después del ingreso con colocación de prótesis de Z-Muller. Recibe profilaxis con dalteparina sódica 5.000 unidades subcutáneas diarias. Una semana después de la intervención comienza con dolor abdominal, vómitos, diarrea y anemización (disminución de $4 \mathrm{~g} / \mathrm{dL}$ en la hemoglobina). Efectuada una ecografía abdominal demuestra la presencia de glándulas suprarrenales con aumento de tamaño patológico, no pudiendo diferenciar si se trata de metástasis o hemorragia. Por presentar deterioro progresivo del nivel de conciencia y en coma (Glasgow 4) se traslada a la UCI. En la analítica practicada destacaba natremia de $110 \mathrm{mEq} / \mathrm{L}$ y cortisolemia $1 \mu \mathrm{g} / \mathrm{L}$. Una tomografía axial computerizada (TAC) craneal demostró un infarto lacunar en el asta frontal del ventrículo izquierdo, y en el EEG existían datos de sufrimiento cerebral muy intenso difuso. La TAC abdominal demostró la presencia de masas suprarrenales bilaterales de $6 \times 7 \mathrm{~cm}$ en el lado derecho y $7 \times 8 \mathrm{~cm}$ en el izquierdo, compatibles con HS, sin poder descartar metástasis (Fig. 1). Con medidas de soporte y tratamiento sustitutivo hormonal, durante su estancia en UCI, se normaliza el nivel de conciencia, siendo trasladada al Servicio de Medicina Interna para completar el estudio. La exploración física no revelaba otros hallazgos de interés. En la analítica presentaba hemoglobina de 12,7 $\mathrm{g} / \mathrm{dL}, 102.000$ plaquetas $/ \mathrm{mm}^{3}$, VSG de $107 \mathrm{~mm}$ en la la hora, APTT de 40,4" (control 32,1"), fosfatasa alcalina $392 \mathrm{U} / \mathrm{L}$, GGT $131 \mathrm{U} / \mathrm{L}$, albúmina $2,8 \mathrm{~g} / \mathrm{dL}$, serología de VHB, VHC y VIH negativas, LDH

Trabajo aceptado: 7 de febrero de 2000

Correspondencia: D. Barreiro García. Servicio de Medicina Interna. Hospital de Cruces. Plaza de Cruces, s/n. 48903 Barakaldo. Vizcaya. 


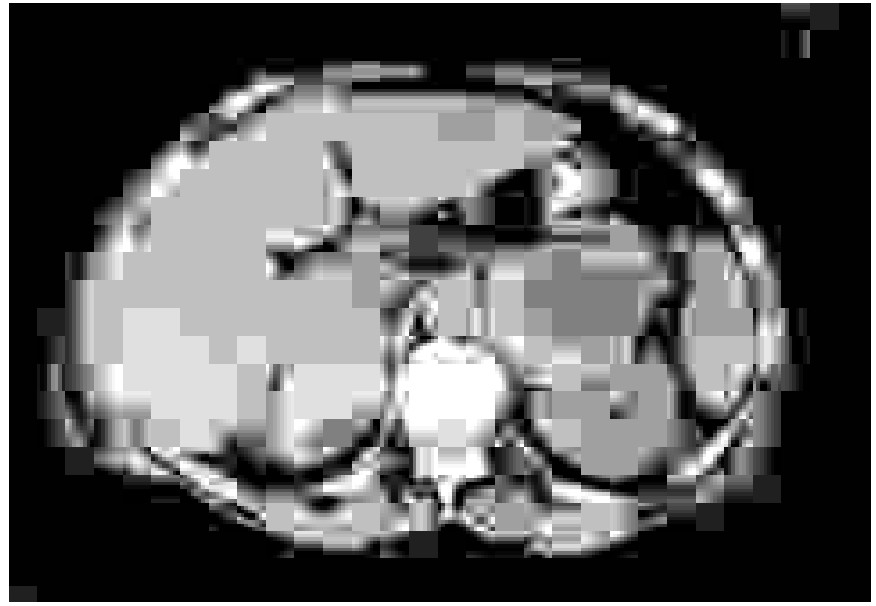

Fig. 1. TAC que muestra suprarrenales agradadas de forma bilateral por hemorragia suprarrenal (HS).

$1.585 \mathrm{U} / \mathrm{L}$, ANA positivos a 1/600, antiDNA negativos, ENA negativos, anticardiolipina negativos, crioglobulinas negativas, C3 y C4 normales, PCR y FR negativos, RPR negativo, anticoagulante lúpico positivo, proteinuria en orina de 24 horas $1 \mathrm{~g}$. Una punción-biopsia dirigida por TAC sobre la suprarrenal izquierda demostró restos hemáticos sin datos de malignidad. Biopsia renal: arquitectura glomerular conservada con fenómenos isquémicos. Resonancia Magnética Nuclear (RMN) abdominal: suprarrenales con un tamaño de $6 \mathrm{~cm}$ y componente hemorragico evidente. ECO Doppler de venas renales sin datos de trombosis.RX de manos sin evidencia de erosiones. Durante su estancia en nuestro servicio presenta trombopenia (nadir de 25.000 plaquetas $/ \mathrm{mm}^{3}$ ), efectuándose aspirado y biopsia medular que demuestra médula ósea con aumento de megacariocitos, que atribuimos a causa autoinmune, y que posteriormente remonta por encima de 100.000 plaquetas $/ \mathrm{mm}^{3}$. Asimismo presentó derrame pleural bilateral (exudado linfocitario esteril con ADA normal) sin datos de embolismo pulmonar en la TAC helicoidal torácica practicada. A pesar de recibir $90 \mathrm{mg} / \mathrm{día}$ de cortisona presenta de nuevo hiponatremia corregida al añadir $0,5 \mathrm{mg}$ de fludocortisona. Con el diagnóstico de HS secundaria a la presencia de anticoagulante lúpico en una paciente portadora de LES no diagnosticado previamente, se inició tratamiento anticoagulante, al principio con heparina sódica iv y posteriormente con dicumarínicos orales, manteniendo el tratamiento sustitutivo hormonal $(30 \mathrm{mg} / \mathrm{día}$ de cortisona y $0,5 \mathrm{mg} /$ día de fludocortisona). En el seguimiento posterior la paciente se encuentra asintomática. Posteriormente los anticuerpos anticardiolipina ( $\operatorname{IgM}, \operatorname{IgG}$ ) se han positivizado en el seguimiento en consultas a títulos medios.

\section{DISCUSIÓN}

En la literatura inglesa se recogen 431 casos en total de HS. Se confirmó la HS postmorten en 341 casos, por TAC en 66 casos, por cirugía en 3 casos y el diagnóstico fue de presunción en 21 casos (6). Como causas desencadenantes se han mencionado situaciones que produzcan estrés, bien médicas o quirúrgicas, la presencia de una diátesis hemorrágica, la diátesis tromboembólica y la terapia con ACTH (6). Además, de todos conocido, el cuadro de HS en el contexto de una sepsis meningocócica, denominado síndrome de Waterhouse Friederichen. En la serie publicada por Rao la HS sucede después de cirugía, quemaduras o trauma en 112 casos. La cirugía más relacionada con HS es la cardiaca a "corazón abierto" y la cirugía traumatológica, sobre todo la colocación de prótesis o reconstrucción, como en nuestro caso $(6,7)$.

En los últimos años se han publicado varios casos de HS asociada a la presencia de anticoagulante lúpico y síndrome antifosfolípido con o sin evidencia clínica de LES acompanante (8-21). Un artículo de revisión reciente recoge 27 casos publicados en la literatura anglosajona (22). Se recogen 14 varones y 13 mujeres, 13 de los cuales presentan otros fenómenos trombóticos y 6 eran portadores de LES. En 10 de estos casos existió un tratamiento previo anticoagulante o antiagregante.

Anatómicamente las glándulas suprarrenales tienen su aporte arterial a través de la arteria frénica inferior, la aorta y las arterias adrenales. Entre 50 y 60 arteriolas provenientes de éstas forman el plexo subcapsular que irriga la zona glomerulosa y se comunica con el plexo de la unión corticomedular. El drenaje sucede por los sinusoides medulares hacia las venas adrenales que tienen un desarrollo muscular notable en su pared. La vena adrenal derecha drena directamente en la cava inferior y la izquierda en la vena renal. La unión corticomedular es la zona más sensible a la hipotensión porque sus células son las últimas a las que llega el aporte nutricio arterial, y precisan una demanda desproporcionada de oxígeno durante el estrés y la estimulación con corticotropina (23). Las glándulas suprarrenales se vuelven vulnerables a la hemorragia por los siguientes factores: el alto flujo sanguíneo de la glándula, dado que todo el aflujo arterial drena por una única vena central y existe una resistencia al drenaje por venospasmo, y por la presencia de una plétora sanguínea provocada por la relativa pobreza del drenaje proveniente del plexo reticular en la unión cortico-medular (24). Se han propuesto varios mecanismos patogénicos en la HS (3):

- ACTH: ya que aumenta de forma notable el flujo arterial de la glándula suprarrenal. Tras una situación aguda como en el caso de una sepsis o un gran quemado, o bien por una situación de estímulo crónico o de tratamiento con ACTH (25).

-Catecolaminas: que contraerían la musculatura de la vena central de drenaje, aumentando la resistencia al flujo y la presión sanguínea local, facilitando así la hemorragia (26).

-Diátesis hemorrágica. Se ha demostrado en animales que la anticoagulación con heparina o dicumarínicos aumenta el riesgo de HS entre 5-10 veces. Se cree que la alta frecuencia de hemorragia en los pacientes tratados con heparina se debe a que se asocia este tipo de anticoagulación a enfermos ingresados en situación de estrés. La trombocitopenia asociada a heparina también se ha relacionado con HS, aunque en la mayoría de los pacientes tratados con heparina no sucedía esta condición $(27,28)$.

-Diátesis trombótica: la trombosis de la vena central de la glándula suprarrenal puede generar HS. Este sería el mecanismo implicado en el SAF (24). No todos los infartos son hemorrágicos ya que se han descrito infartos anémicos o isquémicos.

Siguiendo la excelente revisión de Rao (6) sobre HS con una serie acumulada en la literatura de 277 casos, la clínica viene marcada por los siguientes datos:

1. Dolor: presente en $2 / 3$ de los pacientes, que se supone sucede en el momento de la hemorragia. Puede localizarse en zona lumbar, epigastrio, mesogastrio, flancos, pelvis, o precordio.Un tercio de los pacientes presentan crisis adrenal, por la HS, sin tener dolor. 
2. Fiebre: se detecta en la mitad de los casos. Puede ser continua o intermitente.

3. Clínica abdominal: Se describen datos de peritonismo tan sólo en un $15 \%$.

4. Manifestaciones neuropsiquiátricas: signos atribuibles a insuficiencia suprarrenal como obnubilación, anorexia, debilidad, astenia, sólo sucedieron en el $12 \%$ de los casos.

5. Hipotensión: en el intervalo entre la hemorragia y la crisis adrenal final sólo se detecta hipotensión en el 19\% de los casos.

Los hallazgos de laboratorio recogidos sobre 122 casos de la literatura, siguiendo la misma revisión (6), fueron los siguientes:

1.Hiponatremia, hiperkaliemia y azotemia, atribuidos a insuficiencia suprarrenal en el $56 \%$ de los casos. Hacer notar que casi la mitad no lo tienen.

2.Signos de hemorragia oculta: sobre 90 pacientes con datos fiables, se detecta caída del hematocrito superior al $10 \%$ o disminución mayor de $2 \mathrm{~g} / \mathrm{dL}$ en el $51 \%$.

Antes del desarrollo de la TAC el diagnóstico de HS se hacía postmortem mediante necropsia (21). Las pruebas confirmatorias en la actualidad se basan por una parte en la visualización de la HS por técnicas de imagen, como Ecografía abdominal, TAC o RMN. Las imágenes radiológicas por la TAC en un cuadro de insuficiencia suprarrenal pueden ser diversas (29): a) Atrofia: que puede ser de origen autoinmune, o bien la secuela de HS, tuberculosis y enfermedades granulomatosas; b) Calcificación: típica de la tuberculosis, aunque también puede suceder tras HS, infecciones micóticas y en procesos neoplásicos; c) Agrandamiento: secundario a múltiples causas: tuberculosis de pocos años de evolución, metástasis (pulmón, mama, melanoma...), neoplasias primarias, linfomas, HS recientes, infecciones micóticas y víricas, amiloidosis y sarcoidosis. En nuestro caso la técnica más discriminativa fue la RMN. El patrón radiológico en la TAC de HS es de agrandamiento bilateral, con aumento de la densidad radiológica que evoluciona en el plazo de 6 meses hacia la atrofia con normalización de la densidad y con aparición de calcificacionesen algunos casos (30). En la RMN la HS es hiperintensa en T1, otras lesiones como el linfoma, metástasis y enfermedades granulomatosas aparecen iso o hipointensas con el hígado; en T2 ambos grupos de pacientes muestran imágenes hiperintensas (5).

La confirmación del fracaso adrenal viene dada por la determinación hormonal, mediante un cortisol basal menor de $5 \mu \mathrm{g} / \mathrm{dL}$, que en nuestro caso fue indetectable en plena situación de estres (31) o tras una estimulación corta con $250 \mu \mathrm{g}$ de ACTH, que debe aumentar la cortisolemia $7 \mu \mathrm{g} / \mathrm{dL}$, o bien tener un pico de $20 \mu \mathrm{g} / \mathrm{dL}$ a la hora de la administración de la corticotropina $(3,26)$.

El pronóstico ha mejorado notablemente con el paso del tiempo. Del global de 431 pacientes de la serie de Rao fallece el $80 \%$. Pero si diferenciamos los casos anteriores al año 1980, de 343 pacientes descritos antes sólo sobreviven 9, mientras que de los 88 pacientes posteriores a 1980 sobreviven el $75 \%$ (6). Se ha descrito recuperación de la función endocrina en la HS traumática (33), y en un único caso ocasionado por un anticoagulante lúpico (34). En su mayoría, los pacientes deben recibir tratamiento substitutivo con cortisona indefinidamente, siendo preciso a veces la asociación de 5fludocortisona como en nuestro caso.

En este caso además del antecedente de la cirugía traumatológica, la paciente presentaba datos clínicos y analíticos que sugieren la presencia de un LES (35) y SAF asociado (36). La existencia de insuficiencia suprarrenal como manifestación del SAF aparece ya descrita en los trabajos de Grottolo y posteriores (9). En una revisión sobre 38 pacientes con esta asociación (37) únicamente 7 tenían además un LES. La forma de manifestarse la HS puede ser aguda (21/38 en la serie publicada por Asherson) (37). Aunque rara vez es la forma de debut de la enfermedad (4), suele aparecer en pacientes con SAF ya conocido con manifestaciones trombóticas previas (38).

El riesgo de trombosis recurrente en los pacientes con síndrome antifosfolípido es alto. El mantener una anticoagulación indefinida con un INR mantenido en torno a 3, es deseable en este grupo de enfermos (39). La anticoagulación, dado el evento trombótico que sucede en las venas suprarrenales y que forma parte de la patogenia de la HS, como hemos comentado, no está contraindicada en presencia de la HS. Se debe valorar el riesgo de repetir nuevos episodios de HS en el paciente anticoagulado frente al riesgo de eventos trombóticos en el paciente no tratado (29).

Recintemente a aparecido un excelente artículo de revisión de la hemorragia suprarrenal (HS). (Vella A, Nippoldt TB, Morris JC, Adrenal hemorrhage: A 25-Year experience at the Mayo Clínic. Mayo Clin Proc 2001; 76: 161-168). Se recogen los episodios de HS en la Clínica Mayo durante 25 años, con 141 pacientes. Los casos diagnosticados por los datos clínicos fueron 42, 67 lo fueron tras la necropsin, 30 tras laparotomía, y en 2 casos por punción guiada por TAC como en el que referimos en nuestros artículo. Los casos asociados a síndrome antifosfolípido fueron 12, uno de ellos portedor de LES. La HS fue bilateral con síntomas y signos de insuficiencia suprarrenal en todos estos casos, el 50\% de ellos presentó trombopenia asociada. Los casos postquirúrgicos fueron 14. En 13 de ellos la HS fue bilateral. Los casos relacionados con procedimientos de artroplastia fueron 3. El uso de esteroides sustitutivos cambia dramáticamente el pronóstico vital en la HS. En los casos relacionados al síndrome antifosfolípido sobrevive el $73 \%$ de los tratados frente a ninguno de los no tratados, y en los casos postquirúrgicos sobreviven el $100 \%$ de los tratados y tan sólo el $17 \%$ de los no tratados. Concluyen los autores que el SAF es el factor de riesgo identificable más común en la HS.

\section{Bibliografía}

1. Botteri A, Orell SR. Adrenal hemorrhage and necrosis in the adult. A clicopathological study of 23 cases. Acta Med Scand 1964; 175: 409-419.

2. Russel P. The adrenal glands in shock. Pathology 1972; 4: 5-8.

3. Rao R, H, Vagnucci AH, Amico JA. Bilateral massive hemorrhage. Early recognition and treatment. Ann Intern Med 1989; 110: 227-235.

4. Marie I, Levesque H, Heron F, Cailleux N, Borg JY, Courtois H. Acute adrenal failure secondary to bilateral infarction of the adrenal glands as the first manifestation of primary antiphospholipid antibody syndrome. 1998.

5. Asherson R. Adrenal, hepatic and other intraabdominal manifestations in the antiphospholipid syndrome. En: Asherson RA, Cervera R, Piette 
JCh, Shoenfield Y, eds. The antiphospholipid syndrome. Florida: CRC press 1996; 183-193.

6. Rao R, H. Bilateral massive adrenal hemorrhage. Med Clin North Am 1995; 79: 107-129.

7. Komesaroff PA, Yung AP, Topliss DJ. Postperative primary adrenal failure in a patient with anticardiolipin antibodies. J Rheumatol 1991; 18: 88-90.

8. Eichner H, Schambelan M, Biglieri EG. Systemic lupus erythematous with adrenal insufficiency. Am J Med 1973; 55: 700-705.

9. Grottolo A, Ferrari V, Mariano M et al. Primary adrenal insufficiency, circulating lupus anticoagulant and anticardiolipin antibodies in a patient with multiple abortions and recurrent thrombotic episodes. Haematologica 1988; 73: 517-519.

10. Carett S, Jobin F. Acute adrenal insufficiency as a manifestation of the anticardiolipin syndrome? Ann Rheum Dis 1989; 48: 430431.

11. Asherson R, Hughes GR. Recurrent deep vein thrombosis ans Addison's disease in "primary" antiphospholipid syndrome. J Rheumatol 1989; 16: 378-380.

12. Alperin N, Babu S, Weinstein A. Acute adrenal insufficiency and the antiphospholipid syndrome. Ann Intern Med 1989; 111: 950.

13. Carlisle E, Leslie W. Primary hypoadrenalism in a patient with the lupus anticoagulant. J Rheumatol 1990; 17: 1405-1407.

14. Levy E, Ramsey-Goldman R, Kahl LE. Adrenal insufficiency in two women with anticardiolipin antibodies. Arthritis Rheum 1990; 33: 1842-1846.

15. Walz B, Ho Ping Kong H, Silver R. Adrenal failure and the primary antiphospholipid syndrome. J Rheumatol 1990; 17: 836-837.

16. Lenaerts J, Vanneste S, Knockaertd, Arnout J, vermylen J. SLE and acute Addisonian crisis due to bilateral adrenal hemorrhage: association with the antiphospholipid syndrome. Clin Exp Rheumatol 1991; 9: 407409.

17. McCroskey R, Phillips A, Mott F, et al. Antipospholipid antibodies and adrenal hemorrhage. Am J Hematol 1991; 36: 60-62.

18. Da Costa GM, Forga L, Martínez MS, Orta M. Hemorragia suprarenal ligada a lupus eritematoso sistémico asociado a síndrome antifosfolípido. Rev Clin Esp 1992; 191: 72-73.

19. Ames D, E, Asherson RA, Ayres B, Cassar J, Hughes GR. Bilateral adrenal infarction, hypoadrenalism and aplinter haemorrhages in the "primary" antiphospholipid syndrome. BrJ Rheumatol 1992; 31: 117-120.

20. Molina M, ortega G, Bermudo J, Cuesta F. Insuficiencia suprarrenal asociada a síndrome antifosfolípido primario. An Med Intern 1995; 12: 232-234.

21. Caron P, Chabannier MH, Cambus JP, Fortenfant F, Otal Ph, Suc JM. Definitive adrenal insufficiency due to bilateral adrenal hemorrhage and primary antiphospholipid syndrome. J Clin Endocrinol Metabol 1998; 83: $1437-1439$.

22. Arnason JA, Graziano FM. Adrenal insufficiency in the antiphospholipid antibody syndrome. Semin Arthritis Rheum 1995; 25: 109-116.
23. Dahlberg P, J, Goelher MH, Pehling GB. Adrenal insuficiency secondary to adrenal hemorrhage. Two case reports and a review of causes confirmed by computed tomography. Arch Intern Med 1990; 150: $905-$ 909.

24. Fox B. Venous infarction of the adrenal glands. J Pathol 1976; 119: 6589.

25. Levin TL, Morton E. Adrenal hemorrhage complicating ACTH therapy in Crohn's disease. Pediatr Radiol 1993; 23: 457-458.

26. Szabo S, McComb J, Kovacs K, et al. Adrenocortical hemorrhagic necrosis. The role of catecholamines and retrograde medullary cell embolism. Arch Pathol Lab Med 1981; 105: 536-539.

27. Echaniz A, Diz-Lois F, Pellicer C, Sanmartin J, Suarez Dono I, Pombo F. Hemorragia suprarrenal bilateral, secundaria a tratamiento con heparina: descripción de un caso. An Med Interna 1992; 9: 189-191.

28. Delhumeau A, Moreau X, Chapott C, Houi N, Bigorne JC. Heparinassociated trombocytopenia syndrome: an underestimated etiology of adrenal hemorrhage. Intensive Care Med 1993; 19: 475-477.

29. Sahún M, Villabona C, Montaña E, Fernández Jm, Soler J. Importancia de la tomografía axial computerizada en el diagnóstico etiológico de la enfemedad de Addison. Rev Clin Esp 1992; 190: 177-180.

30. Provenzale JM, Ortel TL, Nelson RC. Adrenal hemorrhage in patients with primary antiphospholipid syndrome: imaging findings. AJR 1995; 165: 361-364.

31. Mattinglyd D, Tyler C. Plasma 11-hydroxicorticosteroid level during stress. Proc R Soc Med 1965; 58: 1010-1012.

32. Grispoon S, Biller BMK. Laboratory assessement of adrenal insufficiency. J Clin Endocrinol Metab 1994; 79: 923-931.

33. Feurstein B, StreetenD. Recovery of adrenal function after failure resulting from traumatic bilateral adrenal hemorrhage. Ann Intern Med 1991; 115: 785-786.

34. Vengrove MA, Amoroso A. Reversible adrenal insufficiency after adrenal hemorrhage. Ann Intern Med 1993; 119: 439.

35. Tam E, Cohen AS, Fries JF, Masi AT, McShane DJ, Rothfield NF, Schaller JG, Talal N, Winchester RJ. The 1982 revised criteria for the classification of systemic lupus erythematous (SLE). Arthritis Rheum 1982; 25: 1271-1277.

36. Harris E. Annotation: Antiphospholipid antibodies. Br J Haematol 1990; 74: 1-9.

37. Asherson R. Hypoadrenalism and the antiphospholipid antibodies. A new cause of "idiopathic" Addison's disease. En Bhatt, James, Besser, Botazzo, Keen, eds. Advances in Thomas Addison's Diseases. Bristol: Journal of Endocrinology Ltd 1994; 87-101.

38. Khamastha M, Font J. Síndrome antifosfolípido. En: J Font, M Kamashta, M Vilardel Eds. Lupus Eritematoso Sistémico. Barcelona: mra, SL 1996; 445-463.

39. Khamashta M, Cuadrado MJ, Mujic F, Taub NA, Hunt BJ, Hughes GR. The management of trombosis in the antiphospholipid-antibody syndrome. N Engl J Med 1995; 332: 993-997. 